\title{
Pratiques enseignantes et " contexte pédagogique » dans le cadre de l'indexation pédagogique de textes
}

\author{
Mathieu Loiseau \\ LIDILEM - Université Stendhal Grenoble 3 \\ mathieu.loiseau@u-grenoble3.fr \\ Georges Antoniadis \\ LIDILEM - Université Stendhal Grenoble 3 \\ georges.antoniadis@u-grenoble3.fr \\ Claude Ponton \\ LIDILEM - Université Stendhal Grenoble 3 \\ claude.ponton@u-grenoble3.fr
}

\section{Indexation pédagogique de textes pour l'enseignement des langues et pratiques}

Bien que la recherche de textes paraisse être une des tâches récurrentes de l'enseignement des langues, il semble que peu d'outils aient été conçus dans l'optique de permettre aux enseignants d'accéder à des supports textuels en fonction de critères relevant de leur problématique (Loiseau et al., 2008). Certains travaux s'intéressent à des aspects particuliers de cette tâche, comme par exemple la recherche de textes en fonction de leur lisibilité (François, 2009), d'autres s'en approchent du fait de traiter de collections de textes, mais concernent un objet d'étude différent : des corpus destinés à être utilisés dans l'enseignement des langues (Braun, 2005; Alcaraz \& Pérez Paredes, 2008). Mais très peu adressent le problème de la recherche de textes pour l'enseignement des langues en tant que tel. Pourtant certains enseignants intègrent cette difficulté à leurs pratiques en se constituant des recueils de textes personnalisés, dont certains sont partagés. Netter (2008), par exemple, réunit des textes pour les enseignants de FLE ou de FLS qu'elle met en ligne, proposant ainsi un fond documentaire adapté à l'enseignement des langues. Cependant son initiative est avant tout un travail d'édition et n'a pas pour objectif de proposer un système pour organiser les textes.

Or, si l'on en croit Roberts et Bosse-Andrieu, « a corpus is only as good as the query system you have to consult it » (Roberts \& Bosse-Andrieu, 2006). Cela ne signifie pas que le contenu d'une base de textes n'a pas d'importance, mais qu'à partir d'une certaine quantité de données, la qualité des textes présents n'est pas une condition suffisante pour que le système soit adapté aux utilisateurs.

Des travaux comme ceux de Sharoff (2008) se sont intéressés plus précisément à la question de l'accès aux ressources. L'approche qu'ils ont adoptée, probablement la plus prompte à donner des résultats, consiste en un recensement des techniques de catégorisation automatique de textes, dont ils tentent ensuite d'utiliser les plus appropriées. Nous nous proposons d'emprunter un chemin différent dont l'objectif est la soumission d'un modèle viable pour l'indexation de textes pour l'enseignement des langues. Selon cette approche la question des outils à utiliser ne peut intervenir qu'après une analyse détaillée des besoins des enseignants.

Les travaux présentés ici s'inscrivent donc dans le cadre de recherches portant sur l'indexation pédagogique de textes pour l'enseignement des langues et plus précisément sur la proposition d'un modèle informatisable adapté à ce problème. Ils s'alimentent des réflexions menées pour la conception de la plateforme MIRTO, au sein desquels nous identifiions une série d'écueils des systèmes d'ALAO. À notre avis, certains de ces écueils découlent directement de la pauvreté du sens associé aux formes dans une bonne partie des logiciels (Antoniadis et al., 2005:65-66). L'approche choisie pour MIRTO consiste en partie à avoir recours à des outils du TAL (Traitement Automatique des Langues) pour accéder à des 
concepts pertinents pour la problématique des enseignants de langue et non uniquement à des chaines de caractères. Outre le rapport de filiation qui existe entre MIRTO et les travaux présentés ici ${ }^{1}$, l'influence des conclusions du premier sur le second sont immédiates. En effet, l'une des idées de départ de ce travail est de considérer les apports potentiels du TAL pour la tâche d'indexation pédagogique de textes. Cependant, avant de nous lancer dans une démarche applicative, il fallait effectuer un travail théorique sur ce qu'était l'indexation pédagogique et ses conséquences sur un modèle pour y parvenir.

Nous définissons l'indexation pédagogique comme une «indexation effectuée selon un langage documentaire permettant à l'utilisateur de rechercher des objets pour les utiliser dans un enseignement » (Loiseau, 2009:15). Dans le cadre de notre travail les objets sont donc des textes et les utilisateurs des enseignants de langue.

Après avoir survolé les principales méthodes d'enseignement des langues et la place qu'y occupe le texte, il nous est apparu que ces méthodes ne constituaient pas un point d'entrée viable pour répondre à notre problème : le recours quasi-universel au texte et l'éclectisme (Puren, 1994) en vigueur (Levy, 1997) rendent la focalisation sur une méthode particulière hazardeuse. En effet, même s'il est possible d'obtenir des statistiques concernant les proportions d'usage des méthodes parmi une population d'enseignants, celles-ci ne rendent pas compte de l'importance de chaque méthode au sein des pratiques de chaque enseignant et encore moins dans le cadre de la recherche de textes. Nous nous sommes alors tournés vers les travaux portant sur la description de ressources pédagogiques en vue de leur échange. L'analyse des principaux standards (LOM (2002), SCORM, IMS LD, etc.) a révélé qu'ils étaient vraisemblablement inadaptés pour la description de ressources brutes ${ }^{2}$ (Pernin, 2006). Il nous est alors paru nécessaire d'adopter une démarche empirique : en vue de vérifier les hypothèses à partir desquelles nous avons écarté les points d'entrée sus-mentionnés, mais aussi, et surtout, pour tenter de comprendre plus avant les pratiques des enseignants quant à la recherche et au choix de textes. Nous présentons dans cet article les études menées et les principales conclusions quant aux pratiques des enseignants et aux propriétés des textes. Nous n'entrerons en revanche pas dans le détail du modèle qui a découlé de ce travail.

\section{Présentation succincte du premier questionnaire}

Nous avons suivi dans le cadre de ce travail une méthodologie classique portant sur des pratiques déclarées $^{3}$ : une série d'entretiens semi-dirigés ${ }^{4}$, suivie d'un questionnaire. Nous avons considéré que notre problème était, à ce stade, analysé dans une perspective compréhensive et explicative pour laquelle l'étude de pratiques déclarées est adaptée (Bru, 2002:70). Ce questionnaire ayant soulevé de nouvelles questions, nous en avons ensuite réalisé un second. Dans cette section, nous présenterons succinctement les objectifs du premier, ainsi que les conclusions les plus directes qui serviront de base à notre réflexion.

\subsection{Objectifs}

L'un des premiers objectifs de ce questionnaire est de valider les remarques qui nous y ont mené, à savoir vérifier qu'au sein d'une population susceptible d'utiliser le système, les enseignants ont effectivement recours à plusieurs méthodes d'enseignement, mais aussi et surtout, nous assurer que du point de vue des enseignants les textes peuvent effectivement être utilisés dans plusieurs contextes tout à fait différents. En effet, c'est l'une des raisons pour lesquelles nous avons considéré les standards de descriptions de ressources pédagogiques inadaptés à notre problème : les propriétés dites pédagogiques permettent avant tout de décrire des activités et ne sont pas des propriétés intrinsèques à des ressources brutes (Recker \& Wiley, 2001), lesquelles semblent pouvoir être utilisées dans des séquences différentes.

Plus que la confirmation de ces hypothèses, qui valident empiriquement des conclusions obtenues par l'analyse de la littérature, le recours à ce questionnaire vise à nous donner un premier accès au processus de recherche de textes, du point de vue le plus général possible. L'objectif serait d'obtenir un certain nombre d'invariants du processus, qui ne soient pas affectés par la langue enseignée, le pays d'exercice ou le public concerné, par exemple. 
Pour ce faire nous circonscrivons notre étude à une partie précise de la planification. Dans la littérature, la planification est vue comme décomposée en trois phases : «[Jackson] distingue la phase préactive ou postactive de la phase interactive : l'enseignant planifie ses cours avant la leçon (préaction) mais aussi entre deux leçons en fonction des leçons qui précèdent (régulation postactive); l'interaction en classe comprend elle-même des opérations cognitives de planification du moment qui suit en fonction de ce qui vient de se passer $^{5}$ » (Tochon, 1989:23). La recherche et le choix de textes appartiennent donc en particulier à la phase de préaction; selon le modèle de Yinger (1978:2), il s'agit même d'un sousproblème de cette phase. C'est en tant que telle que nous voulons l'analyser, non pas seulement en termes de quels critères sont employés pour ces recherches (qui concernent plus directement la suite de notre travail), mais plutôt en termes de quel processus.

Pour y parvenir, nous avons choisi, d'après les résultats de notre étude préliminaire, quelques points d'entrée principaux. Nous nous sommes particulièrement intéressés aux recueils de textes des enseignants et à leur rapport au texte fabriqué. Nous avons également procédé à une analyse croisée des « sources et provenances » des textes. Ici, les sources correspondent aux supports dont les textes sont tirés (ex : périodique) et les provenances aux types de recherche dont ils sont issus. Nous analysons en particulier le recours à la recherche pour une activité donnée et la recherche passive (pratique consistant en le fait de conserver pour l'enseignement des textes rencontrés dans le cadre de lectures personnelles, hors du cadre de la planification). Nous avons enfin considéré le recours aux manuels.

\subsection{Conclusions}

L'objectif de cet article n'est pas de présenter l'étude, mais d'expliciter les conclusions auxquelles nous avons pu arriver grâce aux 130 témoignages recueillis. Nous nous contenterons donc de signaler que si les données recueillies ne nous ont pas permis d'examiner toutes les variables selon l'angle que nous avions prévu, elles nous ont permis de tirer les conclusions suivantes (Loiseau, 2009:188-189) :

- l'une des tâches des enseignants de langue est la recherche de textes ;

- un texte donné peut être utilisé avec des objectifs différents dans des conditions différentes ${ }^{6}$;

- la recherche de textes se fait principalement dans l'optique de les utiliser dans une activité concrète, mais nous avons pu isoler deux autres tendances, ne s'excluant pas mutuellement :

- une forme de recherche passive, qui concerne en premier lieu des enseignants qui possèdent un recueil et qui trouvent fréquemment des textes durant leurs lectures personnelles ;

- des recherches de textes dont l'usage dans l'enseignement des langues est avéré, dont les manuels (ou assimilés) constituent une source privilégiée et qui concerne fréquemment des enseignants se voyant imposer des textes ;

- du point de vue des méthodes employées, les enseignants pratiquent couramment l'éclectisme ${ }^{7}$;

- la langue enseignée (L2), n'a pas d'effet significatif sur le processus général de recherche de textes ${ }^{8}$;

- le cadre dans lequel l'enseignement a lieu n'a pas d'effet significatif sur le processus général de recherche de textes ${ }^{9}$;

- l'expérience de l'enseignant n'a pas d'effet significatif sur le processus général de recherche de textes ${ }^{10}$.

Nous allons maintenant nous appuyer sur ces éléments et d'autres réponses des questionnaires que nous distillerons dans le texte, pour expliciter le processus de recherche et de choix d'un texte.

\section{Flux de la construction d'un texte comme ressource pédagogique}

Du fait des objectifs de nos recherches nous avons adopté, pour parvenir au processus, un point de vue centré sur les ressources (les textes), que nous examinerons de leur écosystème d'origine à leur avatar 
utilisé effectivement comme support d'une activité dans l'enseignement des langues. Pour examiner cette transformation, nous nous appuyons sur la notion de flux du faire de Holloway (2007). Selon lui, un faire est une action humaine et sociale ; humaine, en ce sens que, pour lui, ce qui caractérise l'action humaine réside dans le fait qu'elle s'assortit d'une " projection au delà de ce qui est vers une altérité qui peut être réalisée » (Holloway, 2007:47). L'action de recherche et de choix d'un texte s'inscrit directement dans ce cadre, en tant qu'action de planification. Le faire est également une action sociale qui s'appuie sur toute une série de faires antérieurs, tous indissociables dans un flux global et social du faire. Or, dans le cadre de l'intégration d'un texte à une séquence pédagogique, l'action de l'enseignant s'appuie effectivement pour le moins sur le faire de l'auteur du texte, dont il nous faut expliciter le statut au sein du flux.

\subsection{Objectivations des faires}

«Parler du flux social du faire ne revient pas à nier la matérialité de ce qui est fait. [...] Quand j'écris un livre, il existe en tant qu'objet, il a une existence indépendante de la mienne, et il peut même exister quand je n'existerai plus. En ce sens, on peut dire qu'il y a une objectivation de mon faire subjectif, que ce qui est fait acquiert une existence séparée du faire, qu'il s'abstrait par lui-même du flux du faire. Mais cela n'est vrai que si l'on considère mon action comme un fait individuel. Considérée depuis le flux social de l'agir, l'objectivation de mon faire subjectif devient tout au plus éphémère. [...] L'existence du livre en tant que livre dépend du fait que toi, lecteur, tu le lises, de l'entremêlement entre ton faire (la lecture) et le mien (l'écriture), qui incorpore le fait (le livre) dans le flux social du faire. » (Holloway, 2007:50-51)

De ce fait, les textes présents dans la Figure 1 ne le sont que par les actions d'enseignants, mais avant d'entrer dans le détail celles-ci, nous allons nous intéresser de plus près aux ressources considérées.

Dans notre étude, nous demandions aux enseignants d'ordonner par fréquence leur recours à différentes sources (périodiques, manuels, ouvrages littéraires, Internet, « autres (préciser)»). Les manuels, bien que ne constituant pas la source la plus utilisée des enseignants, concernaient $90 \%$ d'entre eux. La question était de savoir ce qui différenciait les textes issus de manuels des autres, dans la mesure où, comme en témoignent Binon et al. (1998), il est attendu d'un manuel qu'il propose des textes authentiques ${ }^{11}$. Il se peut donc que le même texte, à la mise en forme près, existe dans un périodique et, repris tel quel, dans un manuel. La différence entre les deux provient donc des informations supplémentaires qui l'accompagnent dans le manuel : le fait que le texte soit présent dans le manuel est en soi une indication, mais il sera souvent accompagné de consignes, d'objectifs et autres informations prescriptives que l'enseignant s'appropriera, ignorera ou à partir desquelles il infèrera d'autres propriétés. De notre point de vue, un manuel sera donc un recueil de textes au même titre qu'un périodique, à ceci près qu'il contiendra des informations supplémentaires sur les textes dans l'optique de leur utilisation dans l'enseignement des langues. Cette différence se note dans la Figure 1, par l'inclusion (manuel) ou non (périodique) du texte à la sphère pédagogique, que nous avons représentée en la grisant.

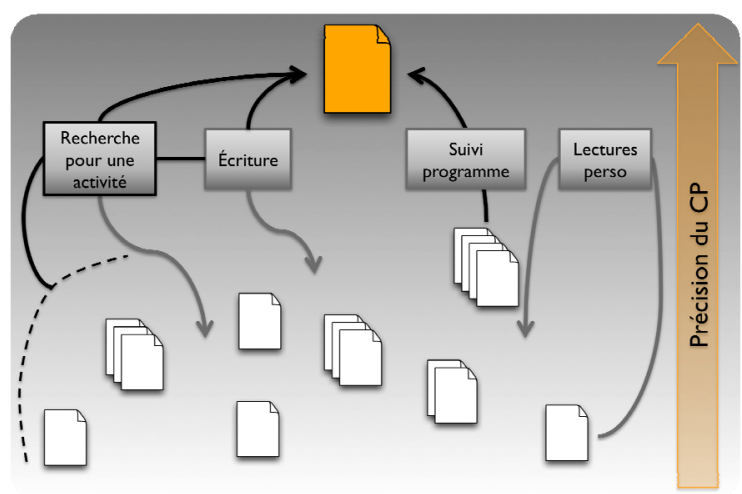

Figure 1 Transformation du texte de son écosystème au statut de texte utilisé dans l'enseignement des langues 


\subsection{Actions}

En effet, la Figure 1 représente une portion du flux du faire, qui, comme nous l'indiquions, est un objet complexe et protéiforme. Ce tronçon de flux correspond donc à l'action potentielle d'un enseignant pour transformer un texte en texte intégré dans une séquence d'enseignement des langues. Le flux avance dans la direction de la flèche orange. Par conséquent les flèches des actions sont à considérer dans l'espace (une action allant contre le flux n'aurait pas de sens). Elles devraient être incluses dans le flux au même titre que les textes, mais cela aurait rendu la figure illisible. Les boîtes et les flèches sont donc des indications extérieures au flux qui vont nous permettre de décrire des actions qui se produisent en son sein. La notion de «sphère pédagogique » permet de se focaliser sur les actions des enseignants tout en les incluant dans un flux global, reconnaissant ainsi leur dépendance vis-à-vis d'autres faires : un texte intégré dans une activité d'enseignement des langues est l'objectivation de l'action de l'enseignant qui l'a planifiée. Mais, avant d'être intégré, le texte a été écrit avec certains objectifs par son auteur, publié dans un certain contexte. Il peut ensuite avoir été ajouté à des archives (dans lesquelles l'enseignant l'aurait trouvé). Chacune de ces étapes correspond aux faires de multiples acteurs, eux-mêmes dépendants d'autres faires.

\subsection{Faires en marge de la problématique de la recherche de textes}

Sans prétention d'exhaustivité ${ }^{12}$, la Figure 1 couvre, d'après les réponses qu'ont pu faire les enseignants, une grande majorité des situations menant à l'utilisation d'un texte en classe; parmi elles, deux concernent des pratiques ne relevant pas directement de la recherche de textes : l'écriture et le fait de suivre le programme (quand celui-ci stipule l'étude d'un texte donné).

\subsubsection{Action d'écriture et texte fabriqué}

Les enseignants peuvent être amenés à écrire eux-mêmes certains des textes qu'ils utilisent en classe. Il s'agit alors de textes dits fabriqués, opposés à l'acception la plus courante de texte authentique (cf. $\left.{ }^{11}\right)$, en ceci qu'ils sont écrits expressément pour être utilisés en classe. Environ $70 \%$ des enseignants ont parfois recours à des textes fabriqués, parmi eux environ $49 \%$ ne font que les rechercher, les autres peuvent également les écrire eux-mêmes. La flèche noire émanant de la boîte «écrire » fait donc référence à ces derniers.

Le fait qu'une très faible proportion des enseignants qui ont recours à des textes fabriqués les écrivent tous $(<4 \%)$ indique qu'au moment de la décision d'avoir recours à un texte fabriqué, celui-ci a souvent déjà été écrit, qu'il soit issu du faire de l'enseignant pour lui-même (texte conservé dans un recueil d'une forme ou d'une autre) ou qu'il s'agisse d'un texte écrit par d'autres (par exemple un texte écrit pour un manuel ou une forme de recueil institutionnalisé ${ }^{13}$ ). La tâche d'écriture peut donc mener à remiser le texte en question (après usage ou non) en attendant qu'il soit ré-inclus au flux par l'action d'un enseignant qui y serait confronté dans ses recherches. Nous plaçons le texte ainsi créé dans la sphère pédagogique (flèche grise), puisque c'est dans l'objectif d'une utilisation en classe qu'il a été écrit.

\subsubsection{Suivi du programme}

Comme certains enseignants se sentaient contraints d'avoir recours à des textes fabriqués (une enseignante déclarait, concernant les raisons qui l'amenait à ne pas utiliser des textes authentiques, être soumise à la pression des parents et des institutions de «faire le livre»), un peu moins de $75 \%$ des enseignants déclarent être soumis à des obligations dans le choix de ressources, sous forme de " textes imposés par le programme ». L'action correspondante dans la Figure 1 est de prendre un texte issu de faires d'acteurs institutionnels (le "programme» peut alors être ramené à une collection de textes appartenant à la sphère pédagogique) pour qu'il devienne un texte utilisé effectivement dans l'enseignement des langues en recherchant une manière de l'intégrer à une séquence pédagogique. D'après la littérature, même si les informations prescriptives atteignent un certain degré de précision, 
l'enseignant se les réappropriera pour les mettre en œuvre en classe (Tochon, 1989:25 ; Dargirolle, $1999: 143-144)$.

\subsubsection{Conséquences sur le diagramme du flux}

Ces deux exemples, bien que les moins représentés au sein des pratiques déclarées des enseignants, nous permettent de mieux appréhender l'adaptation du concept de flux du faire à notre travail et en particulier ses conséquences sur la représentation que nous en avons faite.

Comme nous le signalions, un cas de figure parmi d'autres est celui d'un enseignant qui se verrait imposer l'usage d'un manuel et des textes y figurant (action suivi du programme). Or, les textes que le manuel en question contiendrait peuvent avoir été écrits pour une utilisation dans l'enseignement des langues (action écriture). Par la notion de flux, la Figure 1 rend compte de ce cas. En effet, le flux introduit une notion de temporalité et d'espace, la sphère pédagogique représentée dans la Figure 1 correspond donc à un ensemble de possibles pour un enseignant de langues, à un instant $t$. En outre, ce tronçon (et donc le suivi du programme) est lui-même inclus dans le flux global du faire, chacune de ses actions s'appuie donc sur des faires antérieurs y compris des occurrences antérieures de la «sphère pédagogique » décrite (par exemple l'écriture).

Le schéma doit donc être considéré comme une entité qui se meut et les «boites » comme des étiquettes qui ne sont présentes que par notre description/observation d'un phénomène pendant un laps de temps donné, ce qui signifie qu'à chaque fois qu'une flèche grise réinjecte un texte dans le flux, il pourra être le point de départ d'une occurrence ultérieure du schéma, rendant ainsi compte de la combinatoire complexe des actions, sans pour autant encombrer la figure.

\section{La recherche pour une activité}

La pratique la plus représentée au sein des déclarations des enseignants est la recherche d'un texte en vue de l'utiliser dans une activité spécifique (96,9\% des enseignants). Ce résultat est cohérent avec la théorie. Pour Charlier (1989:31), le matériel pédagogique est une composante de l'activité, il n'est donc pas étonnant qu'elle l'influence. En outre, l'activité occupe une place centrale dans la planification : Clark \& Yinger (1975:15) affirment notamment que les objectifs d'apprentissages sont rarement le point de départ de la planification, qui serait plutôt axée autour des apprenants et des activités. Tochon revient sur ce point de vue et le précise : " [Shavelson \& Stern ont remarqué que] les enseignants n'emploient pas le modèle de [Tyler] qu'on leur a enseigné et qu'ils ne planifient pas par objectifs mais par activités, cellesci s'adaptant mieux aux différents profils d'élèves. » (Tochon, 1989:24)

Pour décrire précisément la recherche en question, nous aurons recours au modèle de Yinger (1978), rapidement évoqué dans la section 2.1. Il définit la planification comme un processus en 3 phases dont la seconde est celle qui contient la recherche de texte (la première concerne la définition du problème, la seconde, sa formulation/solution et la troisième correspond à l'interaction). Cette phase de formulation/solution du problème est effectuée selon la répétition «hélicoïdale » de 3 étapes (Yinger, 1978:36) : élaboration, investigation, adaptation (Charlier, 1989:24).

L'élaboration correspond à la proposition d'une solution à un sous-problème. Elle est évaluée pendant l'étape d'investigation, pour ensuite être adaptée au problème global dans la dernière étape. La répétition de ces étapes doit permettre de préciser graduellement le problème global (planification d'une séquence) pour le résoudre (et le mettre à exécution pendant la phase d'interaction). Nous nous sommes inspirés de ce modèle pour l'adapter au "sous-problème» de la recherche de texte pour une activité, qui est synthétisé dans la Figure 2. L'inscription de la recherche dans le modèle de Yinger indique qu'il peut y en avoir plusieurs itérations pour un seul problème global, nous n'en décrirons qu'une seule. 


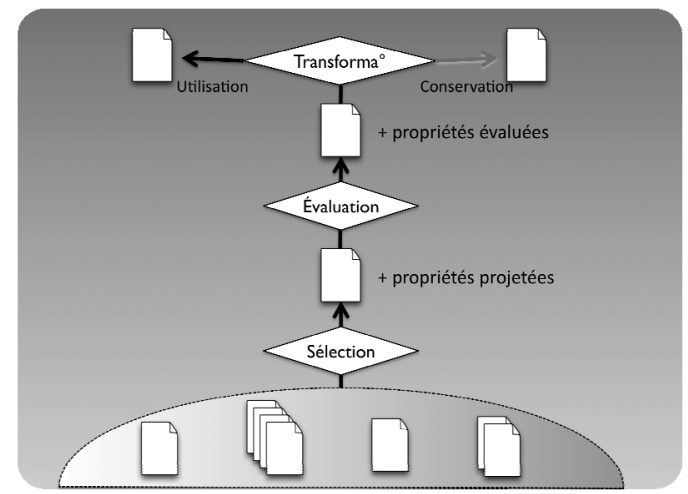

Figure 2 Adaptation du modèle de Yinger à la recherche pour une activité

\subsection{Sélection}

La recherche de texte pour une activité, comme les autres faires de la Figure 1, décrit un processus de transformation d'un texte. Dans le cas de la recherche de texte, celui-ci peut provenir aussi bien directement de la spère pédagogique (par exemple, un texte issu d'un recueil d'enseignant ou d'un manuel $)^{14}$, mais aussi concerner des textes qui n'y figurent pas a priori comme des textes littéraires ou des articles de périodique. Cela a été représenté dans la Figure 1 et la Figure 2 par la zone en pointillés.

Effectuant sa recherche pour une activité précise, l'enseignant se tournera, selon ce qu'il espère trouver, vers une source en particulier. Parmi les témoignages issus de notre étude préliminaire et de ce premier questionnaire, nous pouvons par exemple citer (Loiseau, 2009:207) :

- le choix d'un auteur et d'un type de texte en fonction des structures que l'enseignant attribue à son «style»d'écriture («Roald Dahl, [...] all his short stories are packed with these verbs [...] for emotion and gestures [...], that in French [require] a whole phrase [...]. »);

- le choix d'un journal en fonction de la lisibilité présumée de ses articles (ici le Guardian privilégié pour la clarté de son écriture et malgré ses «coquilles » par rapport à Time et Newsweek jugés trop susceptibles d'utiliser des expressions idiomatiques, de construction parfois douteuse);

- le choix d'un numéro de périodique en fonction d'un type de texte attendu (numéro dominical de journaux sud-américains pour des nouvelles) ;

- le choix d'un type de texte (en l'occurrence fabriqué) par rapport à un type d'activité (exercices lacunaires).

La phase de sélection semble donc s'appuyer sur des propriétés attribuées a priori (présence supposée de structures, lisibilité, type de texte, compatibilité avec un type d'activité) qui sont en lien avec l'activité à mettre en place et que l'enseignant leur attribue en fonction de son expérience et de ses préconceptions nécessaires à cette tâche. Les propriétés peuvent être présumées (lisibilité d'un journal) ou avérées (statut de texte fabriqué) et permettent à l'enseignant de restreindre l'ensemble des textes à considérer. Ces propriétés incluent, par les préoccupations dont elles découlent (liées à l'activité), tout texte à la sphère pédagogique. Elles montrent les mouvements qui existent au sein du flux, mais aussi la dépendance des propriétés au contexte : l'enseignant effectue un choix raisonné qui ne l'oriente pas systématiquement vers la même ressource. Ce choix est influencé par exemple par le public avec lequel il compte utiliser le texte. Le point de vue sur Newsweek et Time de l'enseignante citée ci-dessus serait peut être différent s'il s'agissait de travailler avec des étudiants en école de journalisme (au lieu d'étudiants en sciences par exemple). Elle serait peut être moins sensible aux expressions idiomatiques «non standard» avec un public qui serait plus fréquemment confronté à ce type de textes du fait de sa spécialisation. 
Chez Yinger, cette phase d'élaboration peut être une solution à un sous problème (proposition d'un texte) ou une facette de la définition du problème global (1978:38). Elle s'appuie sur l'exécution de routines mémorisées par l'enseignant ou la tentative d'en définir de nouvelles (1978:37-38).

\title{
4.2 Évaluation
}

La seconde étape s'appelle chez Yinger l'investigation; elle a deux fonctions principales :

\begin{abstract}
«Investigation has two primary functions. First, it provides information about the workability of the elaboration and its success or failure as a subproblem solution. Second, it provides new knowledge and information about the planning problem based on the results of the investigations. » (Yinger, 1978:39)
\end{abstract}

La seconde fonction sort du cadre de la problématique de la recherche, puisque cela correspondrait a l'effet qu'a le choix du texte sur la perception de l'enseignant du problème global. Notre phase d'évaluation correspond en particulier à la première fonction: elle doit répondre à la question de l'utilisabilité du texte dans l'activité planifiée. Il s'agit de l'évaluation du succès ou de l'échec du texte en qualité de support à l'activité. L'évaluation correspond à la confrontation du texte aux besoins de l'enseignant, qui lui assigne donc d'autres propriétés. Il peut s'agir de l'actualisation des propriétés qui lui ont été projeté pendant l'étape de sélection : le texte lu est-il conforme aux attentes de l'enseignant. Il peut s'agir de critères très précis comme le nombre d'occurrences d'une structure grammaticale à travailler (supériorité du nombre d'occurrences donnée comme justification pour l'utilisation d'un texte fabriqué). Mais il peut également s'agir d'autres critères affinés traitant directement de l'adéquation avec d'autres éléments du contexte comme le public (par exemple des critères difficilement formalisables, touchant l'affect, comme «le plaisir, l'intérêt culturel et l'intérêt des élèves », qui est un exemple des préoccupations de près de la moitié des enseignants) ou l'activité en elle-même.

À nouveau, le résultat intermédiaire est une transformation du texte par apposition de nouvelles propriétés, mais, pour l'évaluation, il s'agit de propriétés vérifiées par l'enseignant durant sa lecture.

\subsection{Transformation}

Dans le modèle de Yinger, il s'agit de l'étape d'adaptation, qui vise à formuler et développer le problème global soit en achevant une portion de ce dernier (choix du texte pour nous), soit en obligeant l'enseignant d'adopter un nouveau point de vue sur le problème (1978:42).

Pour nous, l'adaptation du problème à l'évaluation effectuée précédemment consiste à la prise d'une décision que nous pouvons ramener à une nouvelle transformation, qui, cette fois, est l'assignation d'une propriété qui relève du statut du texte, de son utilisation éventuelle dans l'enseignement des langues. Cette décision peut avoir 3 résultats :

- l'assignation d'un contexte d'utilisation en rapport avec le problème initial formulé par l'enseignant (flèches noires dans la Figure 1 et la Figure 2). Le texte va servir de support non seulement à l'activité, mais aussi à la réflexion qui va y mener. La poursuite de cette réflexion peut mener à la «didactisation»du texte: les enseignants déclarent, conformément aux remarques de Vigner (2001:54) ou de Widdowson (1981:93), être amenés à abréger ou adapter le texte ${ }^{15}$ (cf. Figure 1). Le choix peut également être remis en cause par la suite de la planification (ce qui sort du contexte de la recherche de textes);

- le texte est considéré comme inadapté à l'activité en cours de préparation, mais probablement adéquat pour une utilisation par l'enseignant dans un autre contexte. Le texte est remisé dans une forme de recueil, il lui est conferré un ensemble de propriétés plus ou moins précises, qui joueront leur rôle dans le cadre de la sélection d'une recherche ultérieure et seront affinées au cours d'une nouvelle étape d'évaluation (flèches grises dans la Figure 1 et la Figure 2).

- le texte est rejeté. 
Dans ces deux derniers cas la recherche s'est avérée infructueuse et une nouvelle itération de cette séquence en trois étapes est nécessaire, éventuellement en changeant ses critères a priori (ex : recherche d'un texte fabriqué, après la recherche d'un texte authentique). L'enseignant peut même être amené à changer de mode d'obtention en optant pour l'écriture du texte. Il s'agit dans les deux cas de faire adopter à l'enseignant un nouveau point de vue sur le problème (Yinger, 1978:42).

\section{Contexte pédagogique et flux}

Du point de vue du texte, les trois phases détaillées ci-dessus correspondent à la définition graduelle du contexte dans lequel il sera utilisé. En effet, après la formulation du problème (mise en place d'une activité), un texte s'est vu assigner successivement des propriétés projetées, avérées et un changement de statut, toujours affectés par des propriétés de la situation d'enseignement. Nous appellerons ces propriétés elles aussi précisées au fur et à mesure des étapes, le contexte pédagogique. Celui-ci est également intimement lié aux propriétés apposées aux documents, puisque le contexte d'usage du document serait, conformément aux choix des standards, des propriétés associées à la ressource. Cependant, conformément aux déclarations des enseignants concernant les usages multiples des textes, il s'agit de propriétés hautement contextuelles.

Nous définissons cette notion de contexte pédagogique comme «l'ensemble des traits décrivant la situation d'enseignement ». Dans le cadre de l'indexation pédagogique, il n'est pas pertinent de définir toutes les propriétés qui peuvent intervenir dans le contexte pédagogique (CP), mais plutôt de tenter d'identifier les composantes pertinentes du point de vue de la recherche de textes.

Avant de conclure sur l'apport de la notion de contexte pédagogique, il nous faut la confronter aux autres pratiques menant à l'utilisation du texte dans l'enseignement des langues. Nous avons traité le cas de la recherche. Dans le cas de l'écriture, nous pouvons constater que l'enseignant part d'un contexte pédagogique relativement précis et répercute son influence à travers les propriétés du texte qu'il rédige. Le suivi du programme, que nous avions aussi associé à l'action de partir d'un texte et d'arriver à une activité (qui en serait le cas général), correspond donc à définir un contexte pédagogique (une activité) pour un texte donné en s'inspirant ou non de consignes (selon la précision du « programme »).

Enfin, il reste le cas de la recherche passive, que nous n'avons pas détaillé. Il s'agit d'un texte étranger à la sphère pédagogique, puisqu'il a trait aux lectures personnelles de l'enseignant (origine de la flèche dans la Figure 1). Mais, durant sa lecture, l'enseignant finit par lui assigner des propriétés, qui peuvent aller de la simple qualité de potentiellement utilisable dans son enseignement ${ }^{16}$ à des composantes plus précises d'un CP (activité précise, public précis). Dans le cadre de cette action, le contexte pédagogique est également précisé avec la progression dans le flux, jusqu'au point où le texte obtenu au cours d'une lecture personnelle est réinclus dans le flux par le biais d'une action de planification proprement dite : la recherche (l'enseignant part d'un besoin et sélectionne ce texte, par exemple au sein de son recueil, du fait des propriétés qu'il lui a assigné a priori) ou une forme de suivi du programme (l'enseignant part du texte et construit une activité autour).

La notion de contexte pédagogique semble donc adaptée à la description du processus de constitution d'un texte comme ressource pédagogique (au moins pour les actions que nous avons décrites) et a des conséquences directes sur la problématique de l'indexation pédagogique, puisqu'elle atteste de la contextualité des propriétés pédagogiques des textes. Pour avancer dans notre définition d'un modèle pour l'indexation pédagogique de textes, il nous faut comprendre plus précisément le contexte pédagogique, non pas en tentant de définir toutes les propriétés qui peuvent exister au sein du $\mathrm{CP}$, mais en tentant d'isoler les caractéristiques de la situation d'enseignement pertinentes pour la recherche et tenter d'isoler les relations qu'elles entretiennent, entre elles et avec les propriétés intrinsèques des textes. 


\section{Conclusion : le contexte pédagogique comme paradigme}

Suite à la définition de la notion de contexte pédagogique ${ }^{17}$ et aux questions qu'elle a soulevées, nous avons mis en place un second questionnaire qui visait, entre autres objectifs, à établir des exemples de liens entre des aspects du CP et des propriétés intrinsèques aux textes. Nous nous sommes pour cela appuyés sur les réponses apportées au premier questionnaire et avons décidé de croiser :

- le type d'activité (exercice lacunaire de compréhension, d'inférence ou à objectif linguistique, activité de compréhension, introduction de nouvelles notions grammaticales ou de nouveau vocabulaire) avec :

- la taille du texte ;

- le nombre d'éléments représentatifs de la notion (par exemple si la notion est l'utilisation du subjonctif, ce sera le nombre de verbes aux subjonctifs présents dans le texte);

- la tolérance vis-à-vis de la nouveauté (quantité de vocabulaire ou de structures inconnues, différentes de l'objet de la leçon);

- la L1 des apprenants avec la tolérance vis-à-vis de la nouveauté ;

- le niveau des apprenants avec la tolérance vis-à-vis de la nouveauté.

Une séquence de questions est posée aux enseignants qui déclarent avoir recours à chaque type d'activité. Les effectifs fluctuent donc en fonction de la «popularité » de l'activité. Les résultats sont résumés dans la Figure 3, qui contient dans sa partie gauche les aspects du contexte pédagogique considérés et dans sa partie droite les répercussions de ce dernier sur les propriétés des textes. Le schéma représente une séquence descriptive pour un texte donné.

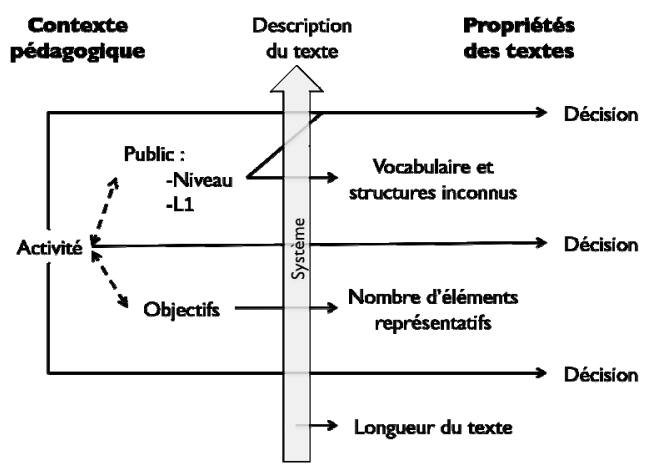

Pour un texte donne

Figure 3 Influence du contexte pédagogique sur l'assignation de propriétés au texte

La longueur du texte est une propriété indépendante du contexte pédagogique : quel que soit l'usage qui est fait du texte, il contiendra le même nombre de mots. Cependant nous avons pu constater que la classe d'activité avait un effet sur la longueur des textes. La décision de juger le texte adéquat ou non en fonction de sa longueur dépendra donc en partie de l'activité ${ }^{18}$.

Le nombre d'éléments représentatifs d'une notion est plus difficile à catégoriser en termes de propriété intrinsèque ou extrinsèque : il relève de l'objet lui-même et devrait être identique quel que soit l'agent qui l'évalue. Cependant son évaluation dépend directement du contexte pédagogique. Sans connaître les objectifs de l'activité, la valeur ne peut être calculée. Ensuite, de la même manière que pour la longueur, une décision est susceptible d'être prise par rapport à cette propriété selon le type d'activité dans laquelle le texte doit être utilisé ${ }^{19}$.

Enfin la variable «tolérance à la nouveauté» nécessite l'évaluation dans le texte de la quantité de vocabulaire et de structures inconnus. Cette propriété fonctionne comme la précédente. Elle dépend du contexte pédagogique (le public et plus particulièrement son niveau) et ne peut être calculée 
indépendamment de cet élément du contexte, mais du point de vue d'un traitement informatique elle est beaucoup plus difficile à mettre en place. Et ce à plus forte raison dans le cadre de la prise de décision qu'elle affecte en fonction du contexte pédagogique. En effet, d'après nos informations, elle dépendra aussi bien du type d'activité (moins grande tolérance pour les activités directement liées à des notions syntaxiques: introduction et exercices lacunaires à objectif linguistique ${ }^{20}$, le niveau du public (la tolérance augmente avec le niveau) et la xénité de la L1 et de la L2 ${ }^{21}$ (la tolérance augmente avec la proximité des langues).

Les données que nous avons recueillies nous ont permis d'établir des relations entre des propriétés extrinsèques (celles du $\mathrm{CP}$ ) et d'autres concernant le texte, dont le statut est plus difficile à définir : elles ne sont pas strictement intrinsèques au texte puisqu'elles varient en fonction du contexte pédagogique ; elles ne sont pas strictement extrinsèques à la ressource non plus puisque, bien que dépendantes d'éléments extérieurs au système, elles ne dépendent pas de l'appréciation de l'enseignant mais sont censées prendre toujours la même valeur pour un texte et un CP donnés. Enfin, la prise de décision dans l'optique de la recherche de textes selon ces propriétés dépend elle aussi des propriétés du contexte pédagogique. Le rôle d'un système d'indexation pédagogique est donc de prendre en compte un ensemble des caractéristiques strictement extrinsèques, celles du $\mathrm{CP}$ en conjonction avec certains éléments des propriétés des textes pour aider l'utilisateur dans la tâche de sélection, espérant ainsi lui proposer un ensemble de textes candidats satisfaisant certains critères. L'enseignant pourra ensuite effectuer son choix parmi cette sélection de textes.

Le modèle que nous proposons, dans les détails duquel nous n'entrerons pas, doit donc considérer les propriétés du textes non comme un ensemble de propriétés figées, mais comme un ensemble de variables dont la valeur est influencée par le contexte pédagogique. Dans le cadre de l'indexation pédagogique, le $\mathrm{CP}$ est donc un «paradigme à la lumière duquel les propriétés des textes prennent leurs valeurs ». C'est dans cette optique que nous proposons d'aborder les propriétés comme des facettes du texte à révéler selon le CP.

Cependant, si nos données sont satisfaisantes pour proposer un modèle, de nombreux travaux sont nécessaires à la mise en place d'un système d'indexation pédagogique de textes pour l'enseignement des langues. La mise en place de chaque critère demande un travail de définition des propriétés faisant intervenir linguistes et didacticiens : parmi les exemples que nous avons cités, la xénité et ses conséquences devrait être formalisées pour ce problème (une piste est offerte par Robert (2004)). D'autres demandent en premier lieu le travail conjoint d'informaticiens et de didacticiens, comme le fait de proposer une modélisation du niveau des apprenants viable dans une perspective de développement d'un outil informatique. L'intégration d'outils TAL semble nécessaire pour l'accès à des concepts du texte, comme, par exemple, les propriétés morphologiques des mots pour évaluer le nombre d'éléments représentatifs d'une structure. Et cela sans perdre de vue qu'il faudra les rendre accessibles à l'utilisateur selon des modalités adaptées à son point de vue d'enseignant. Enfin, des études supplémentaires concernant le détail des relations mises en évidence ici semblent nécessaires. Pour obtenir un outil utilisable par les enseignants et gagner en précision, il faudra s'intéresser aux pratiques constatées et non uniquement aux pratiques déclarées. Un prototype constituerait, via un système de trace, un outil pertinent dans cette optique, mais il ne doit pas être la seule source d'information dans la mesure où nos recherches sur la tâche réalisée par les enseignants pourraient, dans un contexte moins directement lié à l'indexation, être menées selon d'autres critères, mais aussi avec un grain plus fin ${ }^{22}$.

\section{Références bibliographiques}

Alcaraz, J.M. \& Pérez Paredes, P. (2008). What do annotators annotate? An analysis of language teachers' corpus pedagogical annotation. Proceedings of the 8th teaching and language corpora conference (TaLC), Lisbonne, 4-6 juillet 2008, 27-37.

Antoniadis, G., Échinard, S., Kraif, O., Lebarbé, T. \& Ponton, C. (2005). Modélisation de l'intégration de ressources TAL pour l'apprentissage des langues : la plateforme MIRTO. ALSIC, 8 (Numéro spécial TALAL), 65-79. http://alsic.u-strasbg.fr/v08/antoniadis/alsic_v08_04-rec4.htm. 
Binon, J., Geyskens, C. \& Lahousse, K. (1998). Les stratégies de lecture dans quelques manuels de Français Langue Étrangère ou Seconde (FLES) en Flandre. Romaneske, 984, 17-31.

Braun, S. (2005). From pedagogically relevant corpora to authentic language learning contents. ReCALL, 17(1), 4764.

Bru, M. (2002). Pratiques enseignantes : des recherches à conforter et à développer. Revue Française de Pédagogie, $138,63-73$.

Chappelle, C. (2004). Technology and second language learning: expanding methods and agendas. System, 32(4):593-601.

Charlier, É. (1989). Planifier un cours, c'est prendre des décisions. Bruxelles : De Boeck Université.

Charlier, É. \& Charlier, B. (1998). La formation au cœur de la pratique: analyse d'une formation continuée d'enseignants. Bruxelles : De Boeck Université.

Clark, C. \& Yinger, R. (1979). The hidden world of teaching: implications of research on teacher planning. Research Series, 55, http://www.eric.ed.gov/ERICWebPortal/detail?accno=ED175855

Dargirolle, F. (1999). L'évolution de la conception de l'observation de classes en didactique des langues étrangères. Études de Linguistique Appliquée, 4, 114, 141-152.

Holloway, J. (2007). Changer le monde sans prendre le pouvoir: le sens de la révolution aujourd'hui. Paris: Syllepse.

Johns, T. (1986). Micro-concord: a language learner's research tool. System, 14, 2, 151-162.

François, T. (2009). Combining a Statistical Language Model with Logistic Regression to Predict the Lexical and Syntactic Difficulty of Texts for FFL. Proceedings of the EACL 2009 Student Research Workshop, Athènes, 30 mars -2 avril. http://aclweb.org/anthology-new/E/E09/E09-3003.pdf

Levy, M. (1997). Computer-assisted language learning - context and conceptualization. Oxford : Oxford University Press.

Loiseau, M. (2009). Élaboration d'un modèle pour une base de textes indexée pédagogiquement pour l'enseignement des langues (dir. Antoniadis, G. \& Ponton, C.). Thèse soutenue à l’Université Stendhal Grenoble 3.

Loiseau, M., Antoniadis, G. \& Ponton, C. (2008). The concept of "text facet" as a means to achieve pedagogical indexation of a text base dedicated to language teaching. Dans Associação de Estudos e de Investigação Científica do ISLA-Lisboa, éditeurs, Proceedings of the 8th teaching and language corpora conference (TaLC), pages 421-425, Lisbonne, 4 - 6 juillet. http://mathieu.loiseau.free.fr/bdtip/fichiers/articles/Talc8.pdf

LOM (2002). Final 1484.12.1 LOM draft standard document. Document technique, IEEE LTSC WG12.

Netter, C. (2008). ClicNet. (2008). http://clicnet.swarthmore.edu/

Nunan, D. (1989). Designing tasks for the communicative classroom. Cambridge : Cambridge University Press.

Pallotti, G. (2002). La classe dans une perspective écologique de l'acquisition. Acquisition et Interaction en Langue Étrangère, 16.

Pernin, J.P. (2006). Normes et standards pour la conception, la production et l'exploitation des EIAH. Dans Grandbastien, M. \& Labat J.M., éditeurs, Environnements informatiques pour l'apprentissage humain, 201-222. Paris : Hermès et Lavoisier.

Puren, C. (1994). La didactique des langues étrangères à la croisée des méthodes - essai sur l'éclectisme. Essais. Fontenay : Crédif.

Recker, M. \& Wiley, D. (2001). A non-authoritative educational metadata ontology for filtering and recommending learning objects. Interactive Learning Environments, 9, 3, 255-271.

Reffay, C., Chanier, T., Noras, M. \& Betbeder, M.L. (2008). Contribution à la structuration de corpus d'apprentissage pour un meilleur partage en recherche. Sticef, 15(EPAL).

Robert, J.M. (2004). Proximité linguistique et pédagogie des langues non maternelles. Études de Linguistique Appliquée, 4(136):499-511. 
Roberts, R.P. \& Bosse-Andrieu J. (2006). Corpora and translation. Dans Bowker L., éditeur, Lexicography, Terminology, and Translation - Text-based Studies in Honour of Ingrid Meyer:201-214. Ottawa : Presses de l'Université d'Ottawa.

Tagliante, C. (2006). La classe de langue. Paris : CLE International.

Tochon, F. (1989). À quoi pensent les enseignants quand ils planifient leurs cours ? Revue Française de Pédagogie, $86,23-33$.

Sharoff, S., Kurella, S. \& Hartley, A. (2008). Seeking needles in the Web haystack: finding texts suitable for language learners. Proceedings of the 8th teaching and language corpora conference (TaLC), Lisbonne, 4-6 juillet 2008, 268-273.

Vigner, G. (2001). Enseigner le français comme langue seconde. Paris : CLE International.

Widdowson, H.G. (1981). Une approche communicative de l'enseignement des langues. Paris : Hatier-Didier.

Yinger, R. (1978). A study of teacher planning: Description and a model of preactive decision making. Annual meeting of the American Educational Research Association, Toronto, 27-31 mars. http://www.eric.ed.gov/ERICWebPortal/detail?accno=ED152747

\footnotetext{
${ }^{1}$ En effet, le produit du travail présenté ici devait constituer un composant de MIRTO. Il est cependant apparu qu'il était tout à fait cohérent de l'aborder comme un projet à part entière, quitte à l'intégrer ultérieurement à la plateforme.

${ }^{2}$ Par ressources brutes, nous désignons des ressources encore vierges de toute exploitation pédagogique.

${ }^{3}$ La mise en place d'observations visant à analyser des pratiques constatées ou des expérimentations semblait prématurée du fait du peu de données en notre possession.
}

${ }^{4} \mathrm{n}=8$.

${ }^{5}$ Ces trois phases sont largement reprises, par exemple par Yinger (1978:1), Dargirolle (1999:143-144) ou Charlier \& Charlier (1998:16).

${ }^{6} 97,3 \%$ des enseignants déclarent qu'ils considèrent qu'un texte donné peut être utilisé avec des objectifs différents dans des conditions différentes et $94,5 \%$ de ceux-ci (soit $92 \%$ des enseignants interrogés) déclarent l'avoir déjà fait.

${ }^{7} 3,7$ méthodes par enseignant $(\sigma=1,8 ;$ médiane $=4$. 48). $\mathrm{t}(127)=16,85 ; \mathrm{p}<, 001$.

${ }^{8} \Lambda$ de Wilk $=, 798 ; \mathrm{F}(28,398)=, 919 ; \mathrm{p}=, 587$ (test de Box $\left.: \mathrm{p}=, 450\right)$.

${ }^{9} \Lambda$ de Wilk $=, 789 ; \mathrm{F}(35,591)=, 979 ; \mathrm{p}=, 504$ (test de Box $\left.: \mathrm{p}=, 302\right)$.

${ }^{10}$ Pillai : $\mathrm{F}(14,206)=1,417 ; \mathrm{p}=, 147$ (test de Box $\left.: \mathrm{p}=, 003\right)$.

${ }^{11}$ "A rule of thumb for authentic here is any material which has not been specifically produced for the purposes of language teaching. » (Nunan, 1989:54).

${ }^{12}$ Manquent par exemple, le travail sur des textes écrits par les apprenants (Johns, 1986:160-161) ou choisis par ces derniers (Tagliante, $2006: 137$ ).

${ }^{13}$ L'un des témoignages « autres sources » signalait « livre préparé et revisé par un groupe de professeurs du lycée ».

${ }^{14}$ C'est aussi le cas pour le « suivi du programme ».

${ }^{15}$ Suggérant ainsi, pour l'authenticité de la forme du texte, un continuum plutôt qu'une valeur binaire.

${ }^{16}$ Les $2 / 3$ des enseignants ayant recours à la recherche passive $(95 \%$ des enseignants la pratiquent avec plus ou moins de régularité) déclarent qu'ils peuvent les conserver en attendant de trouver une manière adéquate de les utiliser.

${ }^{17}$ Qui n'est pas nouvelle en soi, puisque l'on peut trouver des remarques pointant dans sa direction dans la littérature par exemple dans (Reffay et al., 2008 ; Chappelle, 2004).

${ }^{18}$ ANOVA : $\mathrm{F}=3,362 ; \mathrm{p}<, 05$ (tests post hoc significatifs entre activité de compréhension et les différentes formes d'exercices lacunaires).

${ }^{19}$ ANOVA : $\mathrm{F}=4,739 ; \mathrm{p}<, 005$ (tests post hoc significatifs entre introduction d'une notion et exercices lacunaires de compréhension; et introduction d'une notion syntaxique et exercices lacunaires à objectifs linguistiques). 
Didactique et enseignement, français langue maternelle, français langue seconde DOI $10.1051 / \mathrm{cmlf} / 2010233$

${ }^{20} \chi 2(10)=32,2 ; \mathrm{p}<, 001$.

${ }^{21}$ La notion de xénité, introduite par Weinrich en 1989, est définie par Pallotti de la manière suivante : « la proximité ou l'extranéité d'une langue par rapport à une autre » (Pallotti, 2002).

${ }^{22}$ Un exemple concernerait l'influence de l'expérience sur les types de recherches effectuées par l'enseignant (Loiseau, 2009:194). 